\title{
Production of Bio Fuel Using Green Algea
}

\author{
John Chigbo Onu
}

\begin{abstract}
Hydrogen gas is thought to be the ideal fuel for a world in which air pollution has been alleviated, global warming has been arrested, and the environment has been protected in an economically sustainable manner. Hydrogen and electricity could team to provide attractive options in transportation and power generation. Interconversion between these two forms of energy suggests on-site utilization of hydrogen to generate electricity, with the electrical power grid serving in energy transportation, distribution utilization, and hydrogen regeneration as needed. A challenging problem in establishing $\mathrm{H}_{2}$ as a source of energy for the future is the renewable and environmentally friendly generation of large quantities of $\mathrm{H}_{2}$ gas. Thus, processes that are presently conceptual in nature, or at a developmental stage in the laboratory, need to be encouraged, tested for feasibility, and otherwise applied toward commercialization.
\end{abstract}

Index Terms-Bio fuel, electricity, global warming and Green-Algea.

\section{INTRODUCTION}

Since the pioneering discovery by Gaffron and coworkers over 60 years ago, the ability of unicellular green algae to produce $\mathrm{H}_{2}$ gas upon illumination has been mostly a biological curiosity. Historically, hydrogen evolution activity in green algae was induced upon a prior anaerobic incubation of the cells in the dark. A hydrogenase enzyme was expressed under such incubation and catalyzed, with high specific activity, a light-mediated $\mathrm{H}_{2}$ evolution. The monomeric form of the enzyme, reported to belong to the class of $\mathrm{Fe}$ hydrogenases, [1] M. W. W. Adams and E. I. Stiefel (1990), is encoded in the nucleus of the unicellular green algae. However, the mature protein is localized and functions in the chloroplast stroma. Light absorption by the photosynthetic apparatus is essential for the generation of hydrogen gas because light energy facilitates the oxidation of water molecules, the release of electrons and protons, and the endergonic transport of these electrons to ferredoxin. The photosynthetic ferredoxin (PetF) serves as the physiological electron donor to the Fe-hydrogenase and, thus, links the Fe hydrogenase to the electron transport chain in the chloroplast of the green algae. Under these conditions, the activity of the hydrogenase is only transient (it lasts from several seconds to a few minutes) because, in addition to electrons and protons, the light-dependent oxidation of water entails the release of molecular $\mathrm{O}_{2}$. Oxygen is a powerful inhibitor of the $\mathrm{Fe}$ hydrogenase. Current technological developments in this field have not yet succeeded in overcoming this mutually exclusive nature of the $\mathrm{O}_{2}$ and $\mathrm{H}_{2}$ photoproduction reactions. Thus, the physiological significance and role of the Fe hydrogenase in green algae, which normally grow under aerobic photosynthetic conditions, has long been a mystery. Given the $\mathrm{O}_{2}$ sensitivity of the $\mathrm{Fe}$ hydrogenase and the prevailing oxidative environmental conditions on earth, questions have been asked as to whether the hydrogenase is anything more than a relic of the evolutionary past of the chloroplast in green algae, and whether this enzyme and the process of photosynthesis can ever be utilized to generate $\mathrm{H}_{2}$ gas for commercial purposes. Nevertheless, the ability of green algae to photosynthetically generate $\mathrm{H}_{2}$ gas has captivated the fascination and interest of the scientific community because of the fundamental and practical importance of the process. Below is an itemized list of the properties and promise of photosynthesis in green algal $\mathrm{H}_{2}$ production, and the problems that are encountered with current technology:

1) Photosynthesis in green algae can operate with a photon conversion efficiency of $\geq 80 \%$.

2) Micro-algae can produce $\mathrm{H}_{2}$ photosynthetically, with a photon conversion efficiency of $\geq 80 \%$.

3) Molecular $\mathrm{O}_{2}$ acts as a powerful and effective switch by which the $\mathrm{H}_{2}$ production activity is turned off.

4) This incompatibility in the simultaneous $\mathrm{O}_{2}$ and $\mathrm{H}_{2}$ photoproduction could not be overcome in 60 years of related research.

Aside from the above described photosystem II (PSII)-dependent $\mathrm{H}_{2}$ photo-evolution, which involves water as a source of electrons and produces 2:1 stoichiometric amounts of $\mathrm{H}_{2}: \mathrm{O}_{2}$, an alternative mechanism has been described in the literature. Upon a dark anaerobic incubation of the algae and the ensuing induction of the hydrogenase, electrons for the photosynthetic apparatus are derived upon a catabolism of endogenous substrate and the attendant oxidative carbon metabolism in the green algae. Electrons from such endogenous substrate catabolism feed into the photosynthetic electron transport chain between the two photosystems, and probably at the level of the plastoquinone pool. Light absorption by PSI and the ensuing electron transport elevates the redox potential of these electrons to the redox equivalent of ferredoxin and the hydrogenase, thus permitting the generation of molecular $\mathrm{H}_{2}$. In the presence of the PSII inhibitor 3-(3, 4-dichlorophenyl)-1, 1-dimethylurea (DCMU), this process generates 2:1 stoichiometric amounts of $\mathrm{H}_{2}: \mathrm{CO}_{2}$. Thus, following a sufficiently long dark anaerobic incubation of the culture, initially high rates of $\mathrm{H}_{2}$ production can be detected upon illumination of the algae in the presence of DCMU, a PSII inhibitor.

\section{Two-Stage Photosynthesis AND $\mathrm{H}_{2}$ Production IN GREEN ALGAE}

Recent work has shown that lack of sulfur from the growth 
medium of Chlamydomonas reinhardtii causes a specific but reversible decline in the rate of oxygenic photosynthesis but does not affect the rate of mitochondrial respiration. In sealed cultures, imbalance in the photosynthesis-respiration relationship by $\mathrm{S}$ deprivation resulted in net consumption of oxygen by the cells causing anaerobiosis in the growth medium, a condition that automatically elicited $\mathrm{H}_{2}$ production by the algae. In the course of this recent work, it was shown that expression of the Fe hydrogenase can be induced in the light, so long as anaerobiosis is maintained within the culture. Under such conditions, it was possible to photoproduce and to accumulate significant volumes of $\mathrm{H}_{2}$ gas, using the green alga $C$. reinhardtii, in a sustainable process that could be employed continuously for several days. Thus, progress was achieved by circumventing the sensitivity of the $\mathrm{Fe}$ hydrogenase to $\mathrm{O}_{2}$ through a temporal separation of the reactions of $\mathrm{O}_{2}$ and $\mathrm{H}_{2}$ photoproduction, i.e. by the so-called "two-stage photosynthesis and $\mathrm{H}_{2}$ production" process. The novel application of this two-stage protocol revealed the occurrence of hitherto unknown metabolic, regulatory, and electron transport pathways in the green alga $C$. reinhardtii, leading to the significant and sustainable light-dependent release of $\mathrm{H}_{2}$ gas by the cells. Fig. 1 shows a photograph of an $\mathrm{S}$-deprived, sealed, and $\mathrm{H}_{2}$-producing $C$. reinhardtii culture, in which emanating $\mathrm{H}_{2}$ bubbles are discerned as they are briefly trapped on the walls and neck of the Roux bottle.

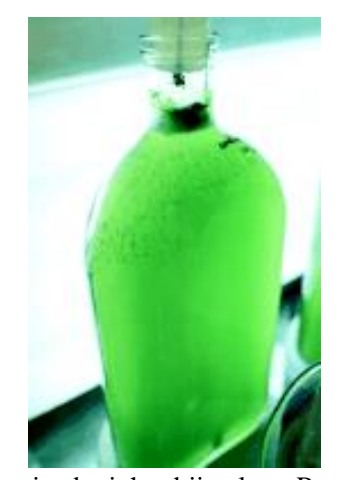

Fig. 1. S-deprived reinhardtii culture Roux bottle.

A hydrogen-producing $C$. reinhardtiiculture. Hydrogen bubbles emanate toward the surface of the liquid medium. The gas is drained through a syringe (inserted in the middle of the silicone stopper) and, through teflon tubing, is collected in an inverted burette and measured by the method of water displacement.

This method may serve as a tool for the elucidation of the green alga hydrogen-related metabolism. Upon further refinement, the method may also serve in the generation of $\mathrm{H}_{2}$ gas for the fuel and chemical industries. The temporal sequence of events in this two-stage photosynthesis and $\mathrm{H}_{2}$-production process is given below:

1) Green algae are grown photosynthetically in the light (normal photosynthesis) until they reach a density of 3 to 6 million cells $\mathrm{mL}^{-1}$ in the culture.

2) Sulfur deprivation is imposed upon the cells in the growth medium, either by carefully limiting sulfur supply in the medium so that it is consumed entirely, or by permitting cells to concentrate in the growth chamber prior to medium replacement with one that lacks sulfur nutrients. Cells respond to this $\mathrm{S}$ deprivation by fundamentally altering photosynthesis and cellular metabolism to survive. [2] A. Akhmanova and B. Boxma, (1998)

3) S deprivation exerts a distinctly different effect on the cellular activities of photosynthesis and respiration. The activity of oxygenic photosynthesis declines quasi-exponentially with a half-time of 15 to $20 \mathrm{~h}$ to a value less than $10 \%$ of its original rate. However, the capacity for cellular respiration remains fairly constant over the $\mathrm{S}$ deprivation period. As a consequence, the absolute activity of photosynthesis crosses below the level of respiration after about $24 \mathrm{~h}$ of $\mathrm{S}$ deprivation. Following this cross point between photosynthesis and respiration, sealed cultures of S-deprived $C$. reinhardtii quickly consume all dissolved oxygen and become anaerobic, even though they are maintained under continuous illumination.

4) Under $S$ deprivation conditions, sealed (anaerobic) cultures ofC. reinhardtii produce $\mathrm{H}_{2}$ gas in the light but not in the dark. The volume and rate of photosynthetic $\mathrm{H}_{2}$ production was monitored from the accumulating $\mathrm{H}_{2}$ gas in an inverted burette, measured from the volume of water displacement. A rate of 2.0 to $2.5 \mathrm{~mL}, \mathrm{H}_{2}$ production $\mathrm{L}^{-1}$ culture $\mathrm{h}^{-1}$ was sustained in the 24 - to 70 -h period. The rate gradually declined thereafter.

5) In the course of such $\mathrm{H}_{2}$ gas production, cells consumed significant amounts of internal starch and protein. Such catabolic reactions apparently sustain, directly or indirectly, the $\mathrm{H}_{2}$ production process. [3] E. S. Bamberger and D. King, (1982).

6) Profile analysis of selected photosynthetic proteins showed a precipitous decline in the amount of Rubisco as a function of time in $\mathrm{S}$ deprivation, a more gradual decline in the level of PSII and PSI proteins, and change in the composition of the light-harvesting complex.

7) Microscopic observations showed distinct morphological changes in $C$. reinhardtii during $\mathrm{S}$ deprivation and $\mathrm{H}_{2}$ production. Ellipsoid-shaped cells (normal photosynthesis) gave way to larger and spherical cell shapes in the initial (0-24 h) stages of S deprivation and $\mathrm{H}_{2}$ production, followed by cell mass reductions at longer (24-120 h) $\mathrm{S}$ deprivation and $\mathrm{H}_{2}$ production times.

A summary of the current state-of-the-art in this field is given below:

1) The absence of sulfur from the growth medium of algae acts as a metabolic switch, one that selectively and reversibly turns off photosynthetic $\mathrm{O}_{2}$ production.

2) In the presence of $S$, green algae do normal photosynthesis (water oxidation, $\mathrm{O}_{2}$ evolution, and biomass accumulation). In the absence of $\mathrm{S}$ and absence of $\mathrm{O}_{2}$, photosynthesis in $C$. reinhardtiislips into the $\mathrm{H}_{2}$ production mode.

3) Reversible application of the switch (presence/absence of $\mathrm{S})$ permits the algae to alternate between $\mathrm{O}_{2}$ production and $\mathrm{H}_{2}$ production (cycling of the stages, thus bypassing the incompatibility and mutually exclusive nature of the $\mathrm{O}_{2}$ - and $\mathrm{H}_{2}$-producing reactions.

4) Interplay between oxygenic photosynthesis, mitochondrial respiration, catabolism of endogenous substrate, and electron transport via the hydrogenase pathway is essential for this light-mediated $\mathrm{H}_{2}$ production 
process.

5) The release of $\mathrm{H}_{2}$ gas serves to sustain baseline levels of chloroplast and mitochondrial electron transport activity for the generation of ATP, which is needed for the survival of the organism under the protracted sulfur deprivation stress conditions (see below).

\section{Physiology OF $\mathrm{H}_{2}$ Production in GReen Algae}

Historically, Hans Gaffron made the first observation of hydrogen metabolism in green algae. [4] L. Florin and A. Tsokoglou, (2001). Upon exposure to hydrogen of anaerobically adapted cells, he observed uptake of molecular $\mathrm{H}_{2}$ by the algae and a concomitant $\mathrm{CO}_{2}$ reduction in the dark. The reverse reaction, e.g. hydrogen production in the light, was first reported with the green alga Scenedesmus obliquus. High rates of $\mathrm{H}_{2}$ evolution could be measured in the light for short periods of time (from several seconds to a few minutes). Electrons were generated either upon the photochemical oxidation of water by PSII, which results in the simultaneous production of $\mathrm{O}_{2}$ and $\mathrm{H}_{2}$, or upon the oxidation of endogenous substrate (Fig. 2), feeding electrons into the thylakoid membrane with the simultaneous release of $\mathrm{CO}_{2}$ to the medium. It is known that $C$. reinhardtii can photoproduce hydrogen when PSII is blocked by DCMU, but no $\mathrm{H}_{2}$ evolution occurs after an addition of 2,5-dibromo-3-methyl-6-isopropyl-p-benzoquinone, which blocks the function of the cytochrome $b-f$ complex. Under anaerobic conditions in the presence of DCMU, accumulated reducing equivalents from the fermentative catabolism of the algae cannot be oxidized via respiration because the terminal electron acceptor $\mathrm{O}_{2}$ is absent. An $\mathrm{NAD}(\mathrm{P}) \mathrm{H}$ reductase protein complex that feeds electrons into the plastoquinone pool recently has been identified in many vascular plant chloroplasts but so far only from the green alga Nephroselmis olivacea. Nevertheless, inhibitor experiments have yielded evidence in support of a thylakoid membrane-localized $\mathrm{NAD}(\mathrm{P}) \mathrm{H}$ reductase in $C$. reinhardtii, suggesting that electrons derived upon the oxidation of endogenous substrate may feed into the plastoquinone pool (Fig. 2). Thereafter, electrons are driven upon light absorption by PSI to ferredoxin. The latter is an efficient electron donor to the $\mathrm{Fe}$ hydrogenase, which efficiently combines these electrons with protons to generate molecularH $\mathrm{H}_{2}$. [5] H. Gaffron.

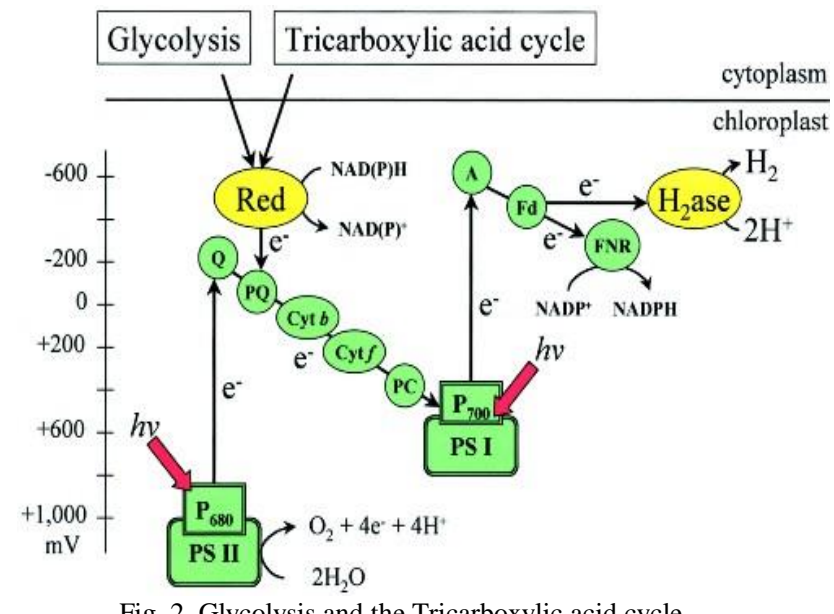

Fig. 2. Glycolysis and the Tricarboxylic acid cycle.
Hydrogenase-related electron transport pathways in green algae. Electrons may originate either at PSII upon photooxidation of water, or at the plastoquinone pool upon oxidation of cellular endogenous substrate (e.g. via glycolysis and the tricarboxylic acid cycle). Electrons in the electron transport chain are transported via PSI to ferredoxin, which serves as the physiological electron donor to the $\mathrm{Fe}$ hydrogenase. $\mathrm{P}_{680}$, Reaction center of PSII; $\mathrm{P}_{700}$, reaction center of PSI; Q, primary electron acceptor of PS II; A, primary electron acceptor of PSI; PQ, plastoquinone; Cyt, cytochrome; PC, plastocyanin; Fd, ferredoxin; Red, $\mathrm{NAD}(\mathrm{P}) \mathrm{H}$ oxido-reductase; $\mathrm{H}_{2}$ ase, hydrogenase; FNR ferredoxin-NADP ${ }^{+}$reductase

The physiology of $\mathrm{H}_{2}$ production upon $\mathrm{S}$ deprivation has many similarities and some distinct differences from the process described above. Sulfur-deprived and sealed cultures of $C$. reinhardtii become anaerobic in the light due to a significant and specific slowdown in the activity of the $\mathrm{O}_{2}$-evolving PSII, which is followed by automatic induction of the Fe hydrogenase and by photosynthetic $\mathrm{H}_{2}$ production. Biochemical analyses revealed that, concomitant with the $\mathrm{H}_{2}$ production process, starch and protein content of the cells gradually declined. Such catabolic pathway(s) could be generating reductant that feeds electrons into the thylakoid membrane, perhaps via a chloroplast NAD(P)H-dependent process. More important, starch catabolism must also generate substrate for the cell's mitochondrial respiration. Mitochondrial respiration scavenges the small amounts of $\mathrm{O}_{2}$ that evolve due to the residual activity of photosynthesis and thus ensures the maintenance of anaerobiosis in the culture (Fig. 3). Thus, the physiology of $\mathrm{H}_{2}$ production by $\mathrm{S}$ deprivation involves a coordinated interaction between:

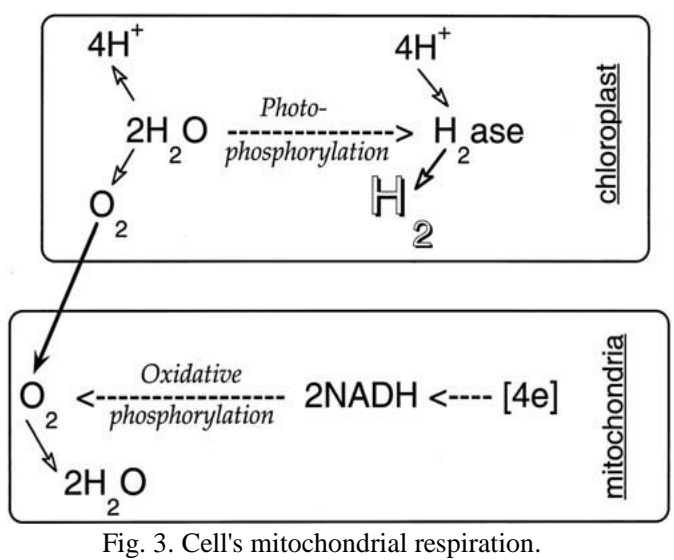

Coordinated photosynthetic and respiratory electron transport and coupled phosphorylation during $\mathrm{H}_{2}$ production. Photosynthetic electron transport delivers electrons upon photo-oxidation of water to the hydrogenase, leading to photophosphorylation and $\mathrm{H}_{2}$ production. The oxygen generated by this process serves to drive the coordinate oxidative phosphorylation during mitochondrial respiration. Electrons for the latter (4e) are derived upon endogenous substrate catabolism, which yields reductant and $\mathrm{CO}_{2}$. Release of molecular $\mathrm{H}_{2}$ by the chloroplast enables the sustained operation of this coordinated photosynthesis-respiration function in green algae and permits the continuous generation of ATP by the two 
bioenergetic organelles in the cell.

1) Oxygenic photosynthesis, i.e. the residual PSII activity for the generation of electrons upon oxidation of water. These electrons are transported through the photosynthetic electron transport chain and eventually feed into the Fe hydrogenase, thereby contributing to $\mathrm{H}_{2}$ production.

2) Mitochondrial respiration scavenges all oxygen generated by the residual photosynthesis and, thus, maintains anaerobiosis in the culture.

3) Endogenous substrate catabolism, including starch, protein, and probably lipid catabolism, yields substrate suitable for the operation of oxidative phosphorylation in mitochondria, and possibly for an NAD(P)H-dependent electron transport in the chloroplast, both of which contribute to the generation of much-needed ATP.

4) Electron transport via the hydrogenase pathway and the ensuing release of $\mathrm{H}_{2}$ gas by the algae sustains a baseline level of photosynthesis and, therefore, of respiratory electron transport for the generation of ATP (Fig. 3) and thus ensures the survival of the organism under protracted stress conditions. [6] R. P. Gfeller and M. Gibbs, (1984)

It is clear that more research is needed to dissect the four-way interplay and the intricate relationships between the processes of oxygenic photosynthesis, mitochondrial respiration, catabolism of endogenous substrate, and electron transport via the hydrogenase pathway leading to $\mathrm{H}_{2}$ production. Nevertheless, the discovery of sustainable $\mathrm{H}_{2}$ production that bypasses the sensitivity of the reversible hydrogenase to $\mathrm{O}_{2}$ is a significant development in the field. It may lead to exploitation of green algae for the production of $\mathrm{H}_{2}$ gas as a clean and renewable fuel. However, the actual rate of $\mathrm{H}_{2}$ gas accumulation was at best $15 \%$ to $20 \%$ of the photosynthetic capacity of the cells, when the latter is based on the capacity for $\mathrm{O}_{2}$ evolution under physiological conditions. The relatively slow rate of $\mathrm{H}_{2}$ production suggests that there is room for significant improvement in the yield of the process, by as much as one order of magnitude. Similarly, other improvements must be made to optimize the process under conditions of mass culture of the algae. For example, optical problems associated with the size of the chlorophyll antenna and the light saturation curve of photosynthesis must be addressed before green algae can achieve high photosynthetic solar conversion efficiencies in mass culture. Moreover, the continuity of the process needs to be addressed because $\mathrm{H}_{2}$ production by $\mathrm{S}$ deprivation of the algae cannot last forever. The yield begins to level off after about $70 \mathrm{~h}$ of $\mathrm{S}$ deprivation. After about $100 \mathrm{~h}$ of $\mathrm{S}$ deprivation, the algae need to go back to normal photosynthesis to be rejuvenated by replenishing endogenous substrate [7] M. L. Ghirardi, J. W. Lee, and T. Flynn.

\section{HOW MUCH $\mathrm{H}_{2}$ CAN ONE EXPECT FROM A MASS Culture of Green Algae?}

Application of the two-stage photosynthesis and $\mathrm{H}_{2}$ production protocol to a green alga mass culture could provide a commercially viable method of renewable hydrogen generation. Table I provides preliminary estimates of maximum possible yield of $\mathrm{H}_{2}$ by green algae, based on the luminosity of the sun and the green algal photosynthesis characteristics. Calculations were based on the integrated luminosity of the sun during a cloudless spring day. In mid-latitudes at springtime, this would entail delivery of approximately $50 \mathrm{~mol}$ photons $\mathrm{m}^{-2} \mathrm{~d}^{-1}$ (Table I, row 1). It is generally accepted that electron transport by the two photosystems and via the hydrogenase pathway for the production of $1 \mathrm{~mol} \mathrm{H}_{2}$ requires the absorption and utilization of a minimum of $5 \mathrm{~mol}$ photons in the photosynthetic apparatus (Table I, row 2). On the basis of these "optimal" assumptions, it can be calculated that green algae could produce a maximum of $10 \mathrm{~mol}(20 \mathrm{~g}) \mathrm{H}_{2}$ per $\mathrm{m}^{2}$ culture area per day. If yields of such magnitude could be approached in mass culture, this would constitute a viable and profitable method of renewable $\mathrm{H}_{2}$ production. [8] M. Gibbs, and C. Chen, (1986).

TABLE I: YIELD OF HYDROGEN PHOTOPRODUCTION BY GREEN ALGAE

\begin{tabular}{|c|c|}
\hline $\begin{array}{l}\text { Photoproduction } \\
\text { Characteristics }\end{array}$ & $\begin{array}{l}\text { Comments on Assumptions } \\
\text { Made }\end{array}$ \\
\hline $\begin{array}{l}\text { Maximum } \\
\text { photosynthetically active } \\
\text { radiation, } 50 \text { mol photons } \mathrm{m}-2 \\
\mathrm{~d}-1 \text { (based on a Gaussian solar } \\
\text { intensity profile in which the } \\
\text { peak solar irradiance reaches } \\
2,200 \mu \mathrm{mol} \text { photons } \mathrm{m}-2 \mathrm{~s}-1 \text { ) }\end{array}$ & $\begin{array}{l}\text { Daily irradiance can vary } \\
\text { significantly depending on season } \\
\text { and cloud cover. It can be greater } \\
\text { than } 50 \text { mol photons } \mathrm{m}^{-2} \mathrm{~d}^{-1} \text { in the } \\
\text { summer and much less than that on } \\
\text { cloudy days and in the winter (Kirk, } \\
\text { 1994). }\end{array}$ \\
\hline $\begin{array}{l}\text { Theoretical minimum photon } \\
\text { requirement for hydrogen } \\
\text { production in green algae: } 5 \mathrm{~mol} \\
\text { photons } / \mathrm{mol} \mathrm{H}_{2}\end{array}$ & $\begin{array}{l}\text { Based on the requirement of } 10 \\
\text { photons for the oxidation of two } \\
\text { water molecules and the release of } \\
\text { four electrons and four protons in } \\
\text { photosynthesis (Ley and Mauzerall, } \\
\text { 1982; Greenbaum, 1988). }\end{array}$ \\
\hline $\begin{array}{l}\text { Theoretical maximum yield } \\
\text { of } \mathrm{H}_{2} \text { production by green algae: } \\
10 \mathrm{~mol} \mathrm{H}_{2} \mathrm{~m}^{-2} \mathrm{~d}^{-1}\left(20 \mathrm{~g} \mathrm{H} \mathrm{H}_{2}\right. \\
\left.\mathrm{m}^{-2} \mathrm{~d}^{-1} ; \sim 80 \mathrm{~kg} \mathrm{H}_{2} \text { acre }^{-1} \mathrm{~d}^{-1}\right)\end{array}$ & $\begin{array}{l}\text { Assuming that all incoming } \\
\text { photosynthetically active radiation } \\
\text { will be absorbed by the green algae } \\
\text { in the culture and that it will be } \\
\text { converted into stable charge } \\
\text { separation. }\end{array}$ \\
\hline
\end{tabular}

However, this optimistic scenario cannot be realized with present day know-how. Three biologically "gray areas" directly impact this $\mathrm{H}_{2}$ production technology. (a) The yield of $\mathrm{H}_{2}$ production currently achieved in the laboratory corresponds to only $15 \%$ to $20 \%$ of the measured capacity of the photosynthetic apparatus for electron transport. (b) The optical properties of light absorption by green algae impose a limitation in terms of solar conversion efficiency in the alga chloroplast. [9] D. Godde and A. Trebst.

This is because wild-type green algae are equipped with a large light-harvesting chlorophyll antenna size to absorb as much sunlight as they can. Under direct and bright sunlight, they could waste up to $60 \%$ of the absorbed irradiance. This evolutionary trait may be good for survival of the organism in the wild, where light is often limiting, but it is not good for the photosynthetic productivity of a green algal mass culture. This optical property of the cells could further lower the productivity of a commercial $\mathrm{H}_{2}$ production farm. [10] E. Greenbaum, (1982) (c) The current necessity to cycle a culture between the two stages (normal photosynthesis in the presence of $\mathrm{S}$ alternating with $\mathrm{H}_{2}$ production upon $\mathrm{S}$ deprivation) introduces a "down time" as far as $\mathrm{H}_{2}$ production is concerned. It is inevitable that the "down time" would 
further erode the yield of the $\mathrm{H}_{2}$ production process. It is clear that these three specific biological challenges $(a-c)$ need to be overcome to effect greater actual yields of green alga $\mathrm{H}_{2}$ production [11] M. L. Ghirardi, J. W. Lee, and T. Flynn.

\section{CONCLUSIONS}

In summary, concerns about global warming and environmental pollution due to the use of fossil fuels, combined with projections of potential fossil fuel shortfall toward the middle of the 21 st century, make it imperative to develop alternative energy sources that are clean, renewable, and environmentally friendly. The recently developed single-organism, two-stage photosynthesis and $\mathrm{H}_{2}$ production protocol with green algae is of interest because significant amounts of $\mathrm{H}_{2}$ gas were generated for the first time, essentially from sunlight and water. Further, this method does not entail the generation of any undesirable, harmful, or polluting byproducts and it may even offer the advantage of value-added products as a result of the mass cultivation of green algae. However, several biological and engineering challenges must be overcome before this promising technology becomes a practical reality. Foremost, the cellular metabolism and basic biochemistry that support this process must be well understood and much fundamental research on the mechanism of $\mathrm{H}_{2}$ production by $\mathrm{S}$ deprivation remains to be done. Ultimately, the advent of hydrogen will bring about technological developments in many fields, including power generation, agriculture, the automotive industry, and other as yet unforeseen applications. It will increase employment, stimulate the economy of all nations on earth, and will have a positive impact on the environment in which atmospheric pollution is all but alleviated and the so-called greenhouse effect is mitigated.

\section{REFERENCES}

[1] M. W. Adam and E. I Stiefel, "The Structure and mechanism of iron-hydrogenes," Biophys Acta, pp. 115-145, 1990.

[2] A. Akhmanova and B. Boxma, "A hydrogenosome with a genome," Nature, vol. 396, pp. 527-528, 1998.

[3] E. S. Bamberger and D. King, "Hydrogen and caborn dioxide evolution by anaerobically adapted chlamydomonas reinhardti," Plant Physiology, pp. 126-127, 1982.

[4] L. Florin, A. Tsokoglou, and T. Happe, "A novel type of Fe.hydrogenenes in the green algae scandesmus obligius is linked to the photosynthetical electron transport chain," Biological Chemistry, pp. 125-132, 1987.

[5] H. Galfrom, "Photosynthesis, photoreduction and dark reduction of carbondioxiade in certain algae," Bio. Rev. Cambridge UK, Phylos, pp. 1-20. 1944.

[6] R. P. Gfeller and M. Gibba, "Fermertative metabolism of chlamydomen as reinhdti," Plant Physiol, ch. 75, pp. 212-218, 1984.

[7] M. Gibbs and C. Chan, Fermentative Metabolism of Clalamydomonas Reinhardti, $3^{\text {rd }}$ ed., Maryland USA, 1986, ch. 82, pp. 160-166.

[8] D. Godde and A. Trebst, "MAOH as electrondonor for the photosynthesis membrane of Chlamydomonas reinhardti," Arch Microbial, pp. 245-252, 1980.

[9] E. Greenbaum, "Photosynthetic hydrogen and oxygen production," Kinetics Studies Science, pp. 879-880, 1982.

[10] E. Greenbaum, "Energetic efficiency of hydrogen photo evolution by algalwatwer-splitting," Biophys, pp. 365-368, 1988.

[11] M. L. Ghirardi, J. W. Lee, and T. Flynn, "A green source of renewable $\mathrm{H}_{2}$," Biotechnol, pp. 506-511, 2000.

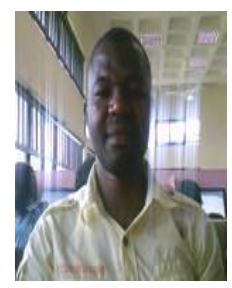

Onu John Chigbo was born at Ezira in Orumber South Local Government Area of Anambra state Nigeria on $4^{\text {th }}$ May 1969. He attended Niger City Primary School and Army Day Secondary School that are both in Onitsha Anambra State Nigeria. He Obtain his B.Eng. degree in agricultural engineering from University of Nigeria Nsukka Enugu, State Nigeria in 1989, and his M.Eng. degree in agricultural engineering form Nnamdi Azikiwe University Awka Anambra State Nigeria in 2013.

He works currently as a lecturer at Federal Polytechnic Oko Anambra State Nigeria.

Engr. Onu is a member of Research and Sustainable Rural Development. 
Electric Energy 
